\title{
Dermatozoenwahn: Psychologische Aspekte für die dermatologische Praxis
}

\author{
Delusional Parasitosis: Psychological Aspects for Dermatological Practice
}

Autoren

Institute
C. Augner ${ }^{1}$, M. Lechner ${ }^{1,2}$, I. Jekel ${ }^{1,2}$

Forschungsinstitut für Grund- und Grenzfragen der Medizin und Biotechnologie am Landeskrankenhaus Salzburg, Universitätsklinikum der Paracelsus Medizinischen Privatuniversität, Salzburg, Österreich

2 Universitätsinstitut für Medizinische Mikrobiologie, Hygiene \& Infektiologie, Landeskrankenhaus Salzburg, Universitätsklinikum der Paracelsus Medizinischen Privatuniversität, Salzburg, Österreich

\section{Bibliografie}

Dol http://dx.doi.org/ 10.1055/s-0030-1255814

Online-Publikation: 8. 10. 2010

Akt Dermatol 2010; 36:

471-473 @ Georg Thieme

Verlag KG Stuttgart · New York ISSN 0340-2541

\section{Korrespondenzadresse}

Dr. Christoph Augner IGGMB - Institut für Grundund Grenzfragen der Medizin Das Gesundheitsforschungsinstitut am LKH Salzburg Universitätsklinikum der PMU Müllner Hauptstraße 48 5020 Salzburg, Österreich c.augner@salk.at

\section{Zusammenfassung \\ $\nabla$}

Dermatozoenwahn ist eine seltene, leicht erkennbare, aber schwer behandelbare psychische Störung. Die betroffenen Patienten gehen dabei fälschlicherweise davon aus von Parasiten, Bakterien und Ungeziefer verschiedenster Art befallen zu sein. Anlaufstellen für die ärztliche Behandlung sind meist Dermatologen, weil sich die

\section{Einleitung \\ $\nabla$}

Der Dermatozoenwahn (auch als Ekbom-Syndrom bezeichnet) gilt als eine wahnhafte Störung, bei der der Patient der festen - aber nachweislich falschen - Überzeugung ist, von Bakterien, Parasiten oder Ähnlichem befallen zu sein. Auch wenn die Krankheit selten ist, hat sie durch die mediale Verbreitung der „Morgellonen-Erkrankung“ an Bedeutung gewonnen. Mit dem Dermatozoenwahn sind Behandler sehr verschiedener Fachrichtungen konfrontiert: Dermatologie, Parasitologie/ Tropenmedizin, Psychologie/Psychiatrie. Besonders wichtig für den Verlauf der Krankheit ist eine funktionierende Zusammenarbeit zwischen diesen Disziplinen.

\section{Typische Symptome \\ $\nabla$}

Charakteristische Symptome für den Dermatozoenwahn (DW) sind Juckreiz und Schmerzen an den „betroffenen“ Hautstellen. In weiterer Folge und bei zunehmender Chronifizierung treten Angstzustände, Selbstverletzungen, Depressionen und soziale Isolation auf. Wichtigstes Kriterium bei DW ist einerseits die anhaltende Überzeugung des Patienten, von Parasiten oder Bakterien befallen zu sein, und andererseits die labordiagnostische Evidenz, dass dies nicht zutrifft. Der behandelnde Arzt gerät hier unweigerlich in einen
„Symptome“ zumeist in der Haut manifestieren. Für den Behandlungserfolg ist das Verhalten des Dermatologen von besonderer Bedeutung, da die meisten Patienten nie einen Psychiater oder Psychologen konsultieren würden. Der vorliegende Beitrag soll einige Hinweise geben, wie trotz der fachfremden Erkrankung ein adäquates und maximal erfolgreiches Management erfolgen kann.

Zielkonflikt. Einerseits ist es nicht möglich, dem Patienten Recht zu geben, andererseits ist Widerspruch häufig das Ende einer Vertrauensbasis. Nicht selten bedeutet dies, dass der Patient den Arzt wechselt (Stichwort: doctor hopping). Dies ist auch der Grund, warum DW oft lange Zeit unbehandelt bleibt.

Ein weiteres besonderes Charakteristikum von DW ist die Selbstverletzung: Anders als die Selbstverletzung bei anderen psychischen Erkrankungen (z.B. Borderline-Syndrom), ist sie bei DW nicht als Abbau von Spannungen zu verstehen, sondern resultiert daraus, dass Personen versuchen in der Haut „Beweismittel“ für den Befall sicherzustellen. Den Parasitologen werden häufig Hautfetzen präsentiert, an denen der Patient kleine Tierchen zu erkennen glaubt. Man spricht in diesem Zusammenhang vom matchbox-sign [1], da die Patienten ihre Funde in Streichholzschachteln für die Untersuchung mitbringen.

\section{Häufigkeit und psychologische Aspelte $\nabla$}

Die Prävalenz von DW ist nicht genau bekannt, sie wird auf 3-7 pro 10000 Personen geschätzt [2]. Altersmäßig ist eine bimodale Verteilung auffällig. Die Spitzen sind dabei zwischen 20 und 30 Jahren sowie bei den über 50-Jährigen. Das Verhältnis Frauen zu Männern beträgt 2:1 [3]. Im Zusammenhang mit der medialen Verbreitung 
der sogenannten „Morgellonen-Erkrankung“ hat der DW an Bedeutung gewonnen. Bei der "Morgellonen-Erkrankung“ sollen Umwelterreger Hautschäden, unspezifische Symptome und sogar kognitive und emotionale Defizite auslösen. Die Verbreitung des Begriffs und der dazu gehörigen Symptome findet hauptsächlich im Internet statt, in der Wissenschaft wird die „MorgellonenErkrankung“ dem DW zugeordnet $[1,4,5]$ Auch wenn DW per definitionem nicht durch biomedizinisch nachweisbare Erreger ausgelöst wird, kann die Erkrankung „ansteckend“ sein: So berichten Frean et al. [6], dass bei 5-15\% der DW-Patienten auch andere Familienmitglieder oder Arbeitskollegen an DW leiden. Wenn ein Patient selbst glaubt befallen zu sein, ist er z.T. auch in der Lage, dies anderen Menschen ebenfalls glaubhaft zu versichern, vor allem wenn die Illusion beinhaltet, dass es sich um etwas Ansteckendes oder Übertragbares handelt. Anhand eines bekannten Beispiels aus der psychologischen Literatur („Arjenyattah-Epidemie“) soll dargestellt werden, wie sich sogar psychische Epidemien über die bekannten Wirkungen des NoceboEffekts hinaus entwickeln können: In einer israelischen Schule klagt ein Mädchen über Atemnot und Schwindel. Weitere Schüler nehmen in der Pause Schwefelwasserstoffgeruch in einer vermutlich defekten Toilette wahr. Daraufhin klagen zehn weitere Personen über Symptome wie Atemprobleme und Bauchschmerzen. Tags darauf lagen 60 Kinder aus der betroffenen Schule im örtlichen Krankenhaus. In den Medien fand dieser Fall Verbreitung (Giftgas, Toxine etc.) und binnen Wochenfrist waren 950 Personen „erkrankt“. Es begann eine fieberhafte Suche nach umweltmedizinischen Ursachen, die aber erfolglos blieb. Nachdem weder Giftgas noch Umweltschäden entdeckt wurden, endete die Epidemie abrupt [7].

DW ist eine Erkrankung, die nicht nur in Einzelfällen zu massiven Selbstverletzungen führen kann, sondern in den meisten Fällen negative Konsequenzen auf Lebensqualität und auf soziale Beziehungen hat. In einer Stichprobe von Boggild et al. [8] zeigte sich, dass DW-Patienten sechs verschiedene Ärzte konsultiert hatten, noch bevor eine adäquate Therapie begonnen wurde.

\section{Diagnose und Behandlung \\ $\nabla$}

Diagnostisch wird zwischen primärem, sekundärem und tertiärem DW unterschieden. Der primäre DW manifestiert sich isoliert, während der sekundäre DW als Folge einer anderen psychischen Störung auftritt, beispielsweise Demenz. Der tertiäre DW ist als Folge anderer medizinischer Zustände zu sehen, wie etwa Drogenmissbrauch oder Durchblutungsstörungen im Gehirn. Tab. 1 stellt die ICD-10-Diagnosen für den DW dar.

Tab. 1 ICD-10-Klassifikation des Dermatozoenwahns.

F06.0 Organische Halluzinose

F22 Anhaltende wahnhafte Störung

DW bleibt häufig lange Zeit unbehandelt. Das hängt vor allem damit zusammen, dass die Patienten die falsche Fachrichtung in Anspruch nehmen: Erste Anlaufstationen sind häufig Dermatologen oder Tropenmediziner. DW-Patienten nehmen gelegentlich auch selbstständig Kontakt mit Parasitologen auf, denen Hautpartikel genauso zur Untersuchung geschickt werden wie Stuhlproben. Hier kann nur festgestellt werden, dass es sich eben um keinen Befall handelt. Die zuständige Berufsgruppe wären Psychologen
Tab. 2 Hinweise für die Behandlung von DW-Patienten.

Zusammenarbeit Dermatologe, Psychologe/Psychiater, Parasitologe/ Mikrobiologe (zumindest in Form von Rücksprache halten)

Investment in Arzt-Patient-Beziehung (Minimalziel: „Patient soll wiederkommen“)

Mikrobiologische Labordiagnostik auch bei Matchbox-Zeichen (Achtung: Ursache muss nicht psychogenetisch sein, auch seltene Allergien möglich)

Vereinbarungen mit dem Patienten treffen, die helfen Selbstverletzungen zu beenden

Keine Gabe von Insektiziden, Antibiotika etc. ohne Indikation

Bei Wahnsymptomatik Gabe von atypischen Antipsychotika, ev. auch Antidepressiva

Konzentration auf Symptome, nicht auf Beweis, dass der Patient falsch liegt

Soziales Umfeld des Patienten miteinbeziehen

Entstandene Hautschäden behandeln

und Psychiater, die von den Patienten - aus ihrer Überzeugung heraus verständlich - gemieden werden. Zum Symptombild gehören die zum Teil gesundheitsschädlichen und auch akut gefährlichen Selbstbehandlungen: z.B. exzessive Hautreinigungen und Einnahme von säurehältigen Substanzen zum „Abtöten des Ungeziefers".

Dem Arzt-Patient-Gespräch kommt bei der Behandlung des DW eine zentrale Bedeutung zu. Hier gibt es keine Patentrezepte. Zunächst geht es darum zu verhindern, dass der Patient den Kontakt abbricht. Das erfordert viel Einfühlungsvermögen durch den Arzt, der die Überzeugung des Patienten weder bestätigen noch ablehnen soll. Ein wesentliches Verhaltensziel ist, dass der Patient aufhört sich selbst zu verletzen. Dazu kann der Arzt versuchen mit dem Patienten eine Vereinbarung zu treffen. Hier kann man eventuell klarmachen, dass selbst bei Parasitenbefall eine solche Maßnahme nichts bringt. Frean et al. [6] empfehlen eine Antipsychotika-Therapie (Erfolgsquote: 50-90\%) zu versuchen, mit der Begründung, dass dadurch Stress und Angst (oder ein „chemisches Ungleichgewicht“) reduziert und so die Lebensqualität verbessert wird. Bei Healy et al. [3] war eine Therapie mit atypischen Antipsychotika sehr erfolgreich. Hier stößt der Arzt an die Grenzen der ethischen Vertretbarkeit bei der Aufklärung über die Erkrankung vs. die beste Behandlung. So ideal die Überweisung an einen Psychiater wäre, wird der Dermatologe häufig aufgrund der Umstände der Behandler bleiben. Rücksprachen mit Psychiatern/Psychologen und Parasitologen sind für die richtige Therapie bei DW besonders wichtig ( Tab. 2).

\section{Schlussfolgerungen}

DW ist eine Erkrankung, die Patienten und Angehörige extrem stark belasten kann. Aufgrund der Ursachenzuschreibung des Patienten werden zumeist Dermatologen mit dieser Störung konfrontiert. Da diese Überzeugung schon Teil der Erkrankung ist, ist die Behandlung so schwierig. Eine Überweisung zum Psychiater kann das Ende der Interaktion bedeuten. Umso wichtiger ist es, dass Dermatologen im niedergelassenen Bereich in die Beziehung mit dem Patienten „investieren“. Die Behandlung sollte in Rücksprache mit Psychologen/Psychiatern und Mikrobiologen erfolgen. 


\section{Abstract}

\section{Delusional Parasitosis: Psychological Aspects for Dermatological Practice \\ $\nabla$}

Delusional parasitosis is a mental disorder that is easy to identify but difficult to treat. Patients have the fixed belief that they are invaded by parasites, bacteria or other bugs. Although it is a mental disorder most of the patients consult dermatologists, because usually „symptoms“ become manifest on the skin. Behavior of the dermatologist is especially important for the success of the treatment, because most of the patients would never consult a psychiatrist or psychologist. This article gives some advices for a successful management of delusional parasitosis despite the different discipline of the illness.

\section{Literatur}

1 Harth W, Hermes B, Freudenmann RW. Morgellons in dermatology. Journal of German Society of Dermatology 2010; 8 (4): 234-242

2 Müller-Spahn F. Diagnostik und Therapie paranoider Syndrome im höheren Lebensalter. Fortschr Neurol Psychiat 2005; 73 (S1): 103 - 106

3 Healy R, Taylor R, Dhoat S et al. Management of patients with delusional parasitosis in a joint dermatology/liaison psychiatry clinic. British Journal of Dermatology 2009; 161: 197-198

4 Lustig A, Mackay S, Strauss J. Letter. Morgellons Disease as Internet Meme. Psychosomatics 2009; 50 (1): 90

5 Wildner M. Kennen Sie Morgellonen? Gesundheitswesen 2009; 71: $795-796$

6 Frean J, De Jong G, Albrecht $R$. Imaginary bugs, real distress: delusional parasitosis. South African Medical Journal 2008; 98 (10): 784- 786

7 Augner C. Psychische Auswirkungen von Mobilfunkstrahlung auf den Menschen. Dissertation. Universität Trier, 2009

8 Boggild AK, Nicks BA, Yen L et al. Delusional parasitosis: six-year experience with 23 consecutive cases at an academic medical center. Int J Infect Dis 2010; 14 (4): 317-321 